\title{
Users' Perceptions of Walkability Attributes in Residential Areas: Reliability and Validity
}

\author{
Yang Ye, Harbin Institute of Technology; China
}

\begin{abstract}
In recent years, urban environmental quality and urban vitality have attracted more scholars' attention than ever as Chinese cities experienced a rapid development stage. To test the influence of urban built environment on walkability in residential areas, this study developed a questionnaire to perceive the users' perception of walkability around their neighbourhood in residential areas, which was based on the Neighbourhood Environment Walkability Scale for Mainland China (NEWS-MC). The original Neighbourhood Environment Walkability Scale (NEWS) are developed for use in USA to measure residents' perceptions of the environment attributes, and has been applied in many countries, such as US, Australia, Korea and Hong Kong, and be modify to be Neighbourhood Environment Walkability Scale for Mainland China (NEWS-MC) and been applied in Chinese cities. NEWS-MC has been found have moderate to high rest-retest reliabilities and good criterion The evidence illustrated high-walkable neighbourhoods be found with higher density, land-use mix, street connectivity, more safety and have more aesthetics elements. In this study, we modified NEWS-MC to reflect the characteristics of cold region Chinese built environment and people's behaviour mode. To perceive the final version of Neighbourhood Environment Walkability Scale for cold region Chinese cities (NEWSCRC), We made a pilot study which include 50 samples, then interview 800 residents from 6 selected residential districts in Harbin (the capital city with highest latitude in China) which were different in walkability attributes and economic status. The final version of the NEWS-CRC included 8 subscales and 3 single items (76 items in total). Test-retest reliability showed moderate to high except 4 items . In total, the NEWS-CRC could illustrate residents' perceptions of walkability attributes in cold region Chinese cities and could be use in other Chinese urban attributes studies related to walking.
\end{abstract}

\section{Keywords}

Keyword 1, keyword 2, keyword 3 (3-5)

\section{Introduction}

The high speed of cities sprawl and urban regeneration worldwide make public space and acclivities in public space gain increasingly attention than ever. Meanwhile, there is growing interest on research about relationship between built environment attributes and residents' physical activity. Studies focus on walking suggest than higher residential density, higher 
level of land use mix- both diversity and accessibility, more aesthetic elements are attributes could hence more walking, and associated with neighbourhoods more walkable .

'Walkability' is referred to and generally defined as "...the extent to which walking is readily available as a safe, connected, accessible and pleasant mode of transportation". In the last decade, there were increasing evidence of the influence of neighborhoods built environment characteristics on walkability. Smith and Clifton illustrated that an built environment friendly to pedestrians usually includes characteristics such as high density, convenience to walk to facilities, system coherence, high street connectivity, safety from traffic and crime, comfortable and attractiveness. Researcher in urban planning and transportation also identified key environment attributes on creating walkable neighborhood. Cervero and Duncan found density, trip distance, land use diversity, existing of sidewalk and bike lane could affect people's choice of walk or bike. The studies by De bourdeaudhuij demonstrated the effects of space proportion, size of block and traffic congestion on user's choice of travel mode .

To make more research on influence of urban environment's walkability on physical activities and psychological health or residents, valid measures of walkability are needed. The Neighborhood Environment Walkability Scale(NEWS) and its abbreviated version(NEWSA) are developed for use in USA to measure residents' perceptions of the environment attributes. They value built environment characteristics that are believed to affect residents' walking choice. Both NEWS and NEWS-A have been found have moderate to high rest-retest reliabilities, and good criterion The evidence illustrated high-walkable neighborhoods be found with higher density, land-use mix, street connectivity, more safety and have more aesthetics elements. Although NEWS and NEWS-A have been applied in many countries, such as US, Australia, Korea and Hong Kong, the validity and reliability of the NEWS or NEWS -A have not been examined in mainland China.

\section{Method}

\subsection{Measures}

Neighborhood Environment Walkability Scale(NEWS) is a instrument used to obtain residents' perception of built environment attributes related to walking and bicycling around where they live. The original NEWS includes 98 items and is rarely used, the abbreviated version of it, NEWS-A , which has similar structure to the full version but has fewer items in subscales, is commonly used and have been applied in many countries (Brazil, Australia, Poland, Korea, Mexico, and Hong Kong). So in this study we also used NEWS-A as the source to develop the NEWS version which appropriate for Mainland China(NEWS-MC). NEWS-A has 8 multi-item subscales, a) residential density; b) land-use mix-diversity; c) Land-use mixaccess; d) street connectivity; e) infrastructure and safety for walking; f) aesthetics; g)traffic hazards and h) Crime safety, and 5 single-item subscales, including Lack of parking, lack of cul-de-sacs, hilliness, physical barriers and social interaction while walking. All items except the ones in residential density and land-use mix-diversity subscales are rated on a 4-point Likert scale(1- strongly disagree, 2-disagree, 3-agree, 4-strongly agree). Items in residential density subscale are rated on 5-point Likeert scale, by asking about the frequency of perceive certain type of residences, and weighted ratings are summed to make a residential 
density perceptions score. Land-use mix-diversity is assessed by time walking from home to various types of destinations, with responses ranging from distance for 1-5mins walking(coded as 5) to distance for more than 30 mins walking(coded as 1 ).

\subsection{Participants and procedures}

2.2.1 Development and adaptation The original NEWS and NEWS-A were developed in US, to develop a version of NEWS which could adapt Chinese cities' build environment attributes and reflects residents' need, 15 experts from multi-research fields, urban planning and urban design(7members), public health(3 members), environment-behaviour studies(2 members), physical activity( 2 members) and Rehabilitation \& Healthcare(1 member) were interviewed with a goal to draft a NEWS-MC. All members of the committee reviewed NEWS-A, we also provided them NEWS-Youth, NEWS for senior citizen and NEWS Hong Kong as reference. Committee members gave their opinions on whether each item should be kept, modified or deleted, or any item should be add after careful thinking about items relevant to mainland Chinese urban environment characteristics and residents' behaviour mode. In total, 57 items in NEWS-A were kept, 9 of them were modified, 17 new items were added and 1 item was deleted considering their relevant to the study setting. (Table 1 ).

Items in residential density subscale were modified to fit the medium to high residential density characteristic in mainland Chinese cities. 'detached single-family residences' and 'townhouses or row hoses of 1-3 stories' were merged into a single item as these kind of residential buildings are very rarely in mainland china; apartments of 4-6, 7-12 and more than 13 stories were modified to apartment of 4-7, 8-18, 19-26 stories and apartments of 27 stories or more was added to this subscale, as 7, 18, 26, 27 and above stories residential buildings are the extreme height for multi-stories, mid-high rise, high rise and super high rise residential buildings through the Chinese building codes. 5 items in land-use mix-diversity were also modified to fit Chinese urban environment(see Table 1). 17 items were added to the questionnaire to provide a better match mainland Chinese urban built environment features and culture background- medium to high population density and building density, high percentage of walking or using public transportation in daily travel, high risk of air pollution and safety issues related by traffic and crime. Item 'video store' was deleted because it is very rarely in mainland Chinese cities now, maybe caused by the development of internet everyone watch or buy movie or video online(see Table 1).

2.2.2 Test-retest reliability Potential participants were chosen from the four Harbin residential neighborhoods mention above, the economic status were obtained from national 2015 Census data. Walkability was measured by residential density, intersection density, destination density( service, commercial and facilities), road density(length of street could walk per km2) and type of street block( square grid, radial shape or have many cul-de-sacs). These data were achieved from baidu map(http://map.baidu.com/).

Four residential neighborhoods, with 125 potential participants were chosen randomly from each neighborhood. The survey were done in March 2017, We went to each neighborhood in both weekday and weekend, morning, noon and evening. When picking the participants, firstly we found the people showed up in that area and asked whether they lived in this neighborhood, if the answer was yes, we further asked whether they would be willing to be reassessed 10 days later after the first survey, and they were told if they finished both survey, they will get $10 \mathrm{RMB}$ as reward. After confirm lived in that residential neighborhood and agreed to attend both survey, one became our participant. The process was the 
interviewer and the participants added each other as 'friend' on wechat (a social App with high popularity in smart phone holders of all ages, add as friends mean two can message each other, and could also pay and receive money, receive and send documents to each other), then sent them the NEWS-MC questionnaire forms, the participants could contacted the interviewer by wechat if they had any questions about the questionnaire. After they sent the first-time forms back, they could get 3RMB. 10 days later we sent them the forms again, and gave who sent forms back 7RMB, the ones who did not sent form back were reminded twice on wechat. The interval between the two surveys ranged from 10-16 days( average 12.4 days) . The recruitment rate of the first survey was $85 \%, 92,93,8580$ and participants responded the second survey, so the total recruitment rate is $70 \%$.

Table 1. Adaptations of the NEWS-A for Mainland Chinese

\begin{tabular}{l}
\hline Item \\
\hline Addition items \\
\hline Distance to community doctor service \\
Distance to Chinese drink or noodle shop \\
Distance to Chinese non-fast food restaurant \\
Distance to Bakery/dessert/cake shop \\
Distance to community centre \\
Distance to copy /scan passport photo-taken shop \\
Distance to place could pay all kinds of fees(gas, water, cable TV and internet) \\
Distance to pets' food or clothes shop \\
Shopping areas are easily accessible via public transport. \\
The streets are so crowded that it is difficult to walk. \\
Need to walk over a pedestrian bridge or through a wide road to access the nearest services \\
There are 'hawkers' and shops on the streets and sidewalks blocking the way \\
There are many cars parking on the sidewalks blocking the way \\
There are indoor, air-conditioned places (shopping malls) where people can walk \\
The streets of my neighborhood are often slippery in winter \\
The level of air pollution in my neighborhood is often high \\
It is unsafe to walk in my neighborhood because of objects dropping from high-rise buildings \\
There are many homeless people, drunk people in my neighborhood \\
\hline Modifications items \\
\hline Two items detached single-family residences and townhouses or row hoses of 1-3 stories \\
merged into a single item \\
apartments of 4-6 stories change to apartment of 4-7 stories. \\
apartments of 7-12 stories change to apartment of 8-18 stories. \\
apartments of more than 13 stories changed to 'apartments of $18-26$ ' stories. Additional item \\
referring to commonness of apartment buildings with more than 26 stories added . \\
Destination 'fruit/vegetable market' renamed 'fresh food market' . \\
Destinations 'fast-food restaurant' renamed 'Chained Western or Chinese fast-food restaurant' \\
Destination 'non-fast food restaurant' changed to 'Western/international non-fast food \\
restaurant' . \\
Destination 'recreation centre' changed to 'recreation centre or outdoor recreation space' . \\
Destination 'clothing store' changed to 'clothing \& shoe store' . \\
'Walkers and bikers on the streets can be easily seen by people in their homes' changed to \\
'walkers and bikers on the streets can be easily seen by other people' . \\
\hline
\end{tabular}


Deleted items

Destination 'video Store'

\section{Results}

Four hundreds and twenty-five participants attended the first survey, with 227 men and 198 women, mean age 45.2. 48\% participants had a collage degree or above. Among all participants, $32 \%$ of them own 1 car or more, and $45 \%$ reported they use public transportation 'very often'. Three hundreds and fifty participants completed both surveys (193 men and 157 woman), response rate was $82 \%$. The sample size per neighborhood was: 90 and 93 in high-walkable neighborhoods, 82 and 85 in low-walkable neighborhoods.

Through previously built satisfaction systems, we took ICC value below 0.50 as poor, 0.50 to 0.75 as moderate, and above 0.75 as reliability. Test-retest reliability for all subscales and single items of the NEWS-MC are shown in Table 2. All subscals and single-items except onehilly roads-showed moderate to good level of reliability $(>0.75), 6$ subscales or single items show high level of reliability $(>0.90)$, and all $p<0.05$.

Table 2 illustrates the results of mean scores on neighborhood walkability subscales between residents from high-walkable and low-walkable neighborhood. Residents from high-walkable neighborhoods provided ratings indicative of higher residential density $(t(412)=6.85, p<0.001)$, land-use mix-diversity $t(420)=-10.97, p<0.001)$; Land-use mix-access ( $\mathrm{t}(420)=4.15, \mathrm{p}<0.001)$; street connectivity $(\mathrm{t}(415)=2.93, \mathrm{p}<0.001)$, infrastructure and safety for walking( $t(397)=5.32, p=0.006)$, traffic hazards( $t(399)=2.07$, $p=0.021)$, parking difficulties $(t(408)=6.81, p=0.004)$, physical barriers $(t(388)=8.74, p<0.001)$ and social interaction while walking $t(386)=-4.85, p<0.001)$. Mean while, residents from low-walkable provided higher ratings of aesthetics $(t(418)=-6.28, p=0.008)$ of their neighborhoods. Residents' perception of crime safety $(t(385)=8.76, p<0.315)$, not have many cul-de-sacs $(t(408)=5.79, p<0.438)$, and hilliness $(t(408)=5.79, p<0.570)$ did not show difference between low and high walkable neighborhoods.

\section{Discussion}

The result of this study indicated the link between walkability and residents' perception of built environment attributes. By using NEWS-MC, a form designed to obtain residents' perception of walkability attributes in medium to high density mainland Chinese cities, as instrument, this study examined the test -retest the reliability and validity of the subscales and single items of NEWS-MC. Moderate to high level of reliability was founded from the result of test-retest reliability of NEWS-MC. The intra-class correlation range from 0.65 to 0.98, which showed a higher reliability than the result in United States(0.58-0.80), Australia $(0,62-0,88)$ and comparable to those observed in Hong Kong(0.57-0.99). The difference may because in US and Australia the researcher used self-administered survey, in this study although we sent the NEWS-MC form to participates but they could communicate with the interviewer by wechat so it kind of like a interviewer administered survey which was used in Hong Kong studyi. Besides, the time interval of first and second survey was 1016 days, which is shorter than the previous studies (14-21 days). 
The result of this study supports the applicability of the NEWS-MC to medium to highdensity cities in mainland China and most Asian cities, such as Harbin. The data showed significant difference in residents' perception on built environment characteristics between high-walkable and low-walkable urban areas. Residential density, land-use mix-diversity, Land-use mix-access, street connectivity, infrastructure and safety for walking, traffic hazards, parking difficulties, physical barriers and social interaction while walking illustrated residents could perceive higher level of attributes in these subscales from high-walkable neighborhoods. The walkability of the neighborhoods was objectively measured by residential density, land-use mix, and pedestrian sidewalk connectivity, and the residents' self-reported result reflected the difference of perceiving these attributes. It is observed that the different direction of ratings on aesthetics, low-walkable neighborhoods were rated higher in most of the items in this subscale, which is the same in Australia and Hong Kong, may be attributed to residents' preference open view and street streets and more green bushes or grass which the low-walkable neighborhoods had more to other aesthetic elements, such as street furniture and sculpture, which the high-walkable neighborhoods had more. The insignificant on crime, hilliness and cul-de-sacs subscales were likely because Harbin is a flat city without many hill and the public order is very good here, and the road network in Harbin is basically grid or radiate, the cul-de-sacs is very rare even in residential area, so residents can not perceive the relationship between items in these subscales or single items and walking.

The mean values comparison between NEWS-MC to NEWS Untied States, Australia and Hong Kong indicated that, as hypothesis, residential density was rated much higher than US and Australia but lower than Hong kong. This can be attributed to the fact that the overall mainland Chinese cities are medium to high density, the urban residential density is higher than US and Australia, but lower than Hong Kong. Land-mix diversity was rated higher in both high-walkable and low-walkable neighborhoods than all the other three studies. It should be notice that the previous studies all used the long version NEWS and we used NEWA the measure instrument, precluded a direct comparison of the findings.

The limitations of this study include:1) The participants are the ones who will to attend the survey, not matched on individual respondent demographic. 2) we only do research in for residential neighborhoods, the small number of areas and small sample number of participants may have limited the reliability and variability of some items. 3) We may omitted some environment attributes that related to walkability of medium to high density mainland Chinese cities.

In conclusion, the result of this study support reliability and construct validity of the NEWSMC, so we could take the NEWS-MC as a instrument for further study of cross-national or international research on the relationship between built environment characteristics and residents' walking choice.

\section{References}

[1] Hardoy, Jorge; Satterthwaite, David (1983) "ISOCARP: Exchange of ideas", Cities, The International Journal of Urban Policy and Planning, Vol. 1 No. 1 (August). 
[2] Humpel, N., Owen, N., \& Leslie, E. (2002). Environmental factors associated with adults' participation in physical activity: a review.American Journal of Preventive Medicine, 22(3), 188-199.

[3] Killingsworth, R. E. (2003). Health promoting community design: a new paradigm to promote healthy and active communities. American Journal of Health Promotion Ajhp, 17(3), 169.

[4] Green, W. A., \& Stoltz, R. R. (2004). Health and community design: the impact of the built environment on physical activity by lawrence $d$. frank, peter engelke and thomas schmid. Landscape \& Urban Planning, 69(4), 467-470.

[5] Sallis, J. F., Bauman, A., \& Pratt, M. (1998). Environmental and policy interventions to promote physical activity. American Journal of Preventive Medicine, 15(4), 379-397.

[6] Lawrence D. Frank, James F. Sallis, Terry L. Conway, James E. Chapman, Brian E. Saelens, \& William Bachman. (2006). Many pathways from land use to health: associations between neighborhood walkability and active transportation, body mass index, and air quality.Journal of the American Planning Association, 72(1), 75-87.

[7] Krizek, K. J. (2000). Pretest-posttest strategy for researching neighborhood-scale urban form and travel behavior. Transportation Research Record Journal of the Transportation Research Board,1722(1722), 48-55.

[8] Clifton, K. J., Smith, A. D. L., \& Rodriguez, D. 2007. The development and testing of an audit for the pedestrian environment. Landscape \& Urban Planning, 80(1-2), 95-110.

[9] Cervero, R., \& Duncan, M. 2011. Walking, bicycling, and urban landscapes: evidence from the san franci. American Journal of Public Health, 93(9), 1478-1483.

[10] De Boudeaudhuij, I., Sallis, J. F., Saelen, B. E., De Bourdeaudhuij, I., Saelens, B., \& De Bourdeaughuij, I., et al. 2003. Environmental correlates of physical activity in a sample of berlgian adults. American Journal of Health Promotion, 18(1), 83-92.

[11] Cerin, E., Conway, T. L., Saelens, B. E., Frank, L. D., \& Sallis, J. F. 2009. Cross-validation of the factorial structure of the neighborhood environment walkability scale (news) and its abbreviated form (news-a).International Journal of Behavioral Nutrition and Physical Activity, 6(1), 1-10.

[12] Leslie, E., Saelens, B., Frank, L., Owen, N., Bauman, A., \& Coffee, N., et al. 2005. Residents' perceptions of walkability attributes in objectively different neighbourhoods: a pilot study. Health \& Place, 11(3), 227-236.

[13]1 Saelens, B.E., Sallis, J.F., Frank, L.D., 2003b. Environmental correlates of walking and cycling: Findings from the transportation, urban design, and planning literatures. Annals of Behavioral Medicine 25 (2), 80 - 91.

[14] Saelens, B. E., Sallis, J. F., Black, J. B., \& Chen, D. 2003. Neighborhood-based differences in physical activity: an environment scale evaluation. American Journal of Public Health, 93(9), 1552.

[15] Cerin, E., Leslie, E., Owen, N., \& Bauman, A. 2008. An australian version of the neighborhood environment walkability scale: validity evidence. Measurement in Physical Education \& Exercise Science,12(1), 31-51. 
[16] Rosenberg, D., Ding, D., Sallis, J. F., Kerr, J., Norman, G. J., \& Durant, N., et al. 2009. Neighborhood environment walkability scale for youth (news-y): reliability and relationship with physical activity. Preventive Medicine, 49(2-3), 213-218.

[17] Shigematsu, R., Sallis, J. F., Conway, T. L., Saelens, B. E., Frank, L. D., \& Cain, K. L., et al. 2009. Age differences in the relation of perceived neighborhood environment to walking. Medicine \& Science in Sports \& Exercise, 41(2), 314.

[18] Cerin, E., Macfarlane, D. J., Ko, H. H., \& Chan, K. C. A. 2007. Measuring perceived neighbourhood walkability in hong kong. Cities,24(3), 209-217. 